\title{
Editorial
}

\section{A favor de la democratización del conocimiento científico para el logro de los objetivos de desarrollo sostenible}

\author{
Ana Lorena Niño Téllez, ${ }^{1}$ \\ Olga Roció Castillo Mayorga, ${ }^{2}$
}

\section{Resumen}

Una sociedad comprometida en la gestión y el acceso libre al conocimiento, logrará la equidad, el bienestar común, la erradicación de la pobreza y todas esas metas propuestas en políticas públicas, como los Objetivos de Desarrollo sostenible (ODS).

El acceso al conocimiento científico, presentado como una de las metas, es esencial e inherente en la investigación, la educación, la toma de decisiones soportadas y la innovación; y como parte de una sociedad debemos reconocer que democratizar el acceso a la información es la clave para la divulgación y el empoderamiento de los profesionales dedicados y comprometidos en el buen uso de conocimiento científico, con el propósito de construir sociedades y ciudadanos competentes para el desarrollo social, económico y cultural, en beneficio del bienestar social y en especial a la superación de las inequidades sociales.

\section{A favor da democratização do conhecimento científico para o alcance dos objetivos de desenvolvimento sustentável}

\section{Sumário}

Uma sociedade comprometida com a gestão e o livre acesso ao conhecimento, alcançará a equidade, o bem-estar comum, a erradicação da pobreza e todas as metas propostas nas políticas públicas, tais como as Metas de Desenvolvimento Sustentável (MDS).

O acesso ao conhecimento científico, apresentado como um dos objetivos, é essencial e inerente à pesquisa, educação, apoio à tomada de decisões e inovação; e como parte de uma sociedade devemos reconhecer que a democratização do acesso à informação é a chave para a disseminação e capacitação de profissionais dedicados e comprometidos com o uso adequado do conhecimento científico, a fim de construir sociedades e cidadãos competentes para o desenvolvimento social, econômico e cultural, para o benefício do bem-estar social e especialmente para superar as desigualdades sociais. 


\section{In favor of the democratization of scientific knowledge to achieve the sustainable development goals}

\begin{abstract}
A society committed to the management and free access to knowledge will achieve equity, common welfare, poverty eradication and all those goals proposed in public policies, such as the Sustainable Development Goals (SDGs).
\end{abstract}

Access to scientific knowledge, presented as one of the goals, is essential and

Los Objetivos de Desarrollo Sostenible (ODS), también conocidos como Objetivos Mundiales, son un llamado universal a la adopción de medidas para poner fin a la pobreza, proteger el planeta y garantizar que todas las personas gocen de paz y prosperidad (UNDP-Programa de las Naciones Unidas para el Desarrollo, 2019a) 17 objetivos transversales orientados a los países que los adoptan, en el cumplimiento de las metas para lograrlos.

El objetivo 16 tiene la meta 16.10 dirigida específicamente en la información, donde es evidente reconocer el conocimiento científico "Garantizar el acceso público a la información y proteger las libertades fundamentales, de conformidad con las leyes nacionales y los acuerdos internacionales"(UNDPPrograma de las Naciones Unidas para el Desarrollo, 2019b).

Es una oportunidad para reconocer que el conocimiento en especial el científico contribuye de manera transversal al inherent in research, education, supported decision making and innovation; and as part of a society we must recognize that democratizing access to information is the key to the dissemination and empowerment of professionals dedicated and committed to the proper use of scientific knowledge, in order to build societies and competent citizens for social, economic and cultural development, for the benefit of social welfare and especially to overcome social inequalities.

mejoramiento de la calidad de vida de los habitantes del planeta, por ello los productores, gestores e intermediarios del conocimiento científico, deben propender por la calidad, la democratización y el acceso libre a la información; a través de estrategias soportadas y con el respaldo de entidades que promuevan y trabajen por el desarrollo del país.

"Los derechos humanos colocan a las personas en el centro del debate. Las respuestas que estén influidas por los derechos humanos, y que los respetan, producen mejores resultados a la hora de vencer a la pandemia, lo cual garantiza una atención sanitaria para todos y preserva la dignidad humana".

América Latina y el Caribe, una de las regiones del mundo con más necesidades económicas, de educación, protección social, pobreza y falta de información en salud; cuenta con el modelo Biblioteca Virtual de Salud (BVS) que funciona, como estrategia y plataforma de cooperación 
para la gestión de la información y conocimiento descentralizada en los países de la región, con la coordinación de BIREME, desarrolla metodologías y se apropia del trabajo colaborativo con el fin de apoyar la investigación, la academia y la toma de decisiones en salud soportadas.

En el año 2018 se realizó la Séptima reunión de Coordinación Regional de la Biblioteca Virtual en Salud (BVS7) y la 10a․ Edición del CRICS10, tuvo como tema "Hacia los logros de la Agenda 2030: aportes de la evidencia y del conocimiento". Un foro presencial para el intercambio de conocimiento entre la red de productores, intermediarios y usuarios de la información técnico científica en la región de América Latina y el Caribe. En la reunión de coordinaciones nacionales, para este congreso, se destacóel "Fortalecimiento de las redes de colaboración" con el fin de promover una discusión sobre modelos de trabajo colaborativo para el desarrollo del modelo BVS, alineadas hacia el alcance de los Objetivos de Desarrollo Sostenible de la Agenda 2030. Un espacio de intercambio de experiencias y mejores prácticas donde las Bibliotecas Virtuales (regional, nacionales y temáticas) y las redes de colaboración más destacadas, aportan al fortalecimiento de la Red de cooperantes, a la visibilidad de información de calidad en salud producida por la comunidad académica y científica; siendo así, una de las reuniones más importante a nivel Internacional en información y comunicación científica en salud, que durante este periodo adopta los ODS como parte del trabajo para presentar y debatir el estado del arte regional e internacional en esta temática.

Para la clausura de la reunión se realizó el lanzamiento oficial de la Declaración de São Paulo a favor de la Democratización del Conocimiento Científico para el logro de los Objetivos de Desarrollo Sostenible que se convierte en la ahora de la ruta de la región para el logro de los objetivos en especial los siguientes ODS:

- ODS 3: Asegurar una vida saludable y promover el bienestar para todos y todas, en todas las edades

- ODS 9: Aumentar en forma significativa el acceso a la tecnología de la información y la comunicación y esforzarse por facilitar el acceso universal y asequible a Internet en los países menos adelantados a más tardar en 2020.

- ODS 16: “Asegurar el acceso público a la información y proteger las libertades fundamentales, de conformidad con las legislaciones nacionales y los acuerdos internacionales".

A continuación, encuentran los principales apartes de la declaración en texto completo:

\section{Nosotros,}

Integrantes del Sistema Latinoamericano y del Caribe de Información en Ciencias de la Salud, reunidos en la Séptima Reunión de Coordinación Regional de la Biblioteca Virtual en Salud (BVS7) y en el Décimo Congreso Regional de Información en Salud (CRICS10),

\section{Considerando:}

- Los Objetivos de Desarrollo Sostenible de la Agenda 2030 y de la Agenda de Salud Sostenible para las Américas 2018-2030 (ASSA2030) que concentran, respectivamente a los deseos de los países en nivel mundial y regional;

- Que el conocimiento científico es esencial e inherente al avance de la investigación, la educación y las innovaciones metodológicas y tecnológicas; 
- Que el acceso y la divulgación del conocimiento científico abierto empodera a los investigadores $\mathrm{y}$ profesionales comprometidos con el bien común;

- Que el acceso a la evidencia basado en el conocimiento científico relevante y actualizado empodera a las sociedades y a los ciudadanos para el desarrollo social, económico y cultural, en beneficio del bienestar social y en especial a la superación de las inequidades sociales;

- Quelas políticas públicas y los tomadores de decisión necesitan de información precisa, relevante y actualizada para que sus acciones estén basadas en la coproducción con actores científicos, profesionales y sociales;

- Que las tecnologías digitales facilitan la democratización del acceso a la información y el mejoramiento sistemático y continuo de la salud;

- La creciente demanda de la sociedad de sistemas de salud robustos, efectivos, accesibles y de intervención basados en la evidencia, que contemplan, cuando sea apropiado, la medicina tradicional y complementaria científicamente comprobada.

\section{Instamos a:}

Los dirigentes, autoridades, investigadores y demás profesionales de los países y territorios de América Latina y el Caribe, así como a la Organización Panamericana de la Salud (OPS) y a su Centro Latinoamericano y del Caribe de Información en Ciencias de la Salud (BIREME), los sistemas nacionales de salud y las instituciones de investigación, educación y atención a la salud, a:

1. Reconocer la importancia de la intersectorialidad y la relación entre el conocimiento y la información científica, tecnológica y tradicional, para que los sistemas de salud sean inclusivos y respondan a las necesidades de todas las personas, de una manera integral;

2. Incluir en sus programas, proyectos y acciones estratégicas, la promoción y el uso de información y conocimiento científico abierto, para fortalecer en todos los niveles las políticas regionales y nacionales de salud;

3. Formular las políticas y tomar decisiones con base en evidencias científicas, disponibles en los sistemas de información en salud;

4. Desarrollar capacidades de generación, transferencia y uso de la evidencia y del conocimiento en salud, promoviendo la investigación, la innovación y el uso de las tecnologías;

5. Alinear las estrategias y prácticas de cooperación técnica en información científica y tecnológica entre países e instituciones, con los programas regionales y nacionales, los Objetivos de la Agenda 2030 y ASSA2030;

6. Considerar la visión y los principios de la Ciencia Abierta en el desarrollo de las estrategias y prácticas de la cooperación técnica en información científica y tecnológica en salud;

7. Considerar la visión y los principios de la ciencia ciudadana en la planificación, evaluación, producción y divulgación científica y tecnológica en salud;

8. Promover políticas, programas y acciones de formación de los sistemas nacionales de información científica, en buenas prácticas de gestión de información y conocimiento; 
9. Reconocer y promover la Biblioteca Virtual en Salud (BVS), el índice de la Literatura Latinoamericano y del Caribe en Ciencias de la Salude (LILACS) y los Descriptores en Ciencias de la Salud (DeCS), como metodologías e instrumentos de acceso libre, visibilidad y calidad de la información, la producción científica $\mathrm{y}$ tecnológica en salud; $\mathrm{y}$

10. Ampliar la colaboración nacional e internacional entre actores sociales que apoyan la BVS, LILACS y DeCS, por medio de programas de formación y desarrollo técnico, liderado por BIREME para la creación de productos, servicios, metodologías y tecnologías de captura, recolección, registro, preservación, publicación y diseminación de la información científica y técnica, en los sistemas nacionales de educación, investigación y atención a la salud.

Ciudad de São Paulo en el 4 de diciembre de 2018.

\section{Conclusiones}

La declaración de Sao Paulo, a favor de la democratización del conocimiento científico para el logro de los objetivos de desarrollo sostenible, hace parte de un modelo colaborativo en los países de América Latina y el Caribe, ofreciendo un beneficio global, con el propósito de transformar socialmente a la comunidad y generarestrategias deuso parala tecnología, información y la comunicación; a través de la interacción en la distancia, el intercambio de información, almacenamiento de datos en la nube, transmisión de conocimiento y la difusión de avances científicos. Una guía que debería ser apropiada por todos los actores que intervienen en la gestión de la información: productores, intermediarios y usuarios; el avance tecnológico abre nuevas fronteras, cierra brechas y establece nuevos retos para alfabetización de la comunidad.

En el marco de la Pandemia ha tenido un fuerte impacto para la Agenda 2030, ya que nunca antes había sido tan importante reducir la brecha digital y buscar la equidad en el acceso a las tecnologías de la información, es lamentable que todavía existen lugares sin conexión a Internet; sin poder acceder a la educación en línea y a la comunicación, se hace urgente que tengan la oportunidad y acceso abierto a la información para lograr los objetivos comunes.

\section{Bibliografía}

BIREME OPS/OMS. (2018). CRICS10. Retrieved from Declaración de São Paulo a favor de la Democratización del Conocimiento Científico para el logro de los Objetivos de Desarrollo Sostenible website: https://www.crics10. org/declaracion-de-sao-paulo-a-favor-de-lademocratizacion-del-conocimiento-cientificopara-el-logro-de-los-objetivos-de-desarrollosostenible/

UNDP-Programa de las Naciones Unidas para el Desarrollo. (2019a). Objetivos de Desarrollo Sostenible. Retrieved from Objetivos de Desarrollo Sostenible website: https://www. undp.org/content/undp/es/home/sustainabledevelopment-goals.html

UNDP-Programa de las Naciones Unidas para el Desarrollo. (2019b). Objetivos de Desarrollo Sostenible. Retrieved from Objetivo 16: Paz, Justicia e Instituicones Sólidas website: https:// www.undp.org/content/undp/es/home/ sustainable-development-goals/goal-16-peacejustice-and-strong-institutions.html 